University of Wollongong

Research Online

Faculty of Engineering and Information

Faculty of Engineering and Information

Sciences - Papers: Part B

Sciences

2019

Microstructure and water distribution in catalysts for polymer electrolyte fuel cells, elucidated by contrast variation small-angle neutron scattering

Satoshi Koizumi

Ibaraki University

Satoru Ueda

Ibaraki University

Takumi Inada

Ibaraki University

Yohei Noda

Ibaraki University

Robert A. Robinson

University of Wollongong, Ibaraki University

Follow this and additional works at: https://ro.uow.edu.au/eispapers1

Part of the Engineering Commons, and the Science and Technology Studies Commons

Research Online is the open access institutional repository for the University of Wollongong. For further information contact the UOW Library: research-pubs@uow.edu.au 


\title{
Microstructure and water distribution in catalysts for polymer electrolyte fuel cells, elucidated by contrast variation small-angle neutron scattering
}

\author{
Abstract \\ By using small-angle neutron scattering (SANS) reinforced by scanning electron microscopy, the fine \\ structure of catalysts for polymer electrolyte fuel cells has been investigated. The experimental data \\ resulting from contrast variation with mixed light and heavy water (H2O/D2O) are well described by a \\ core-shell model with fluctuations in concentration between water and Nafion. In particular, SANS \\ obtained with the mixed water ratio $30 / 70$, which corresponds to a matching point between mixed water \\ and Nafion, shows a broad scattering maximum, which is attributed to a $5 \mathrm{~nm}$-thick Nafion shell on the \\ surface of the larger carbon particles. After swelling by water, the ionomer layer absorbs water at the 17 \\ wt\% level. By changing the H2O/D2O ratio, it was further confirmed that the catalyst with the ionomer \\ exhibits water repellence, whereas the bare catalyst without the ionomer is wetted by water. Because it is \\ very difficult to extract more information, for instance regarding the Pt-Nafion interactions, by means of \\ small-angle scattering, reflectometry and grazing-incidence scattering experiments with neutrons should \\ be attempted on a model catalyst prepared on a flat substrate.

\section{Disciplines} \\ Engineering | Science and Technology Studies

\section{Publication Details} \\ Koizumi, S., Ueda, S., Inada, T., Noda, Y. \& Robinson, R. A. (2019). Microstructure and water distribution in \\ catalysts for polymer electrolyte fuel cells, elucidated by contrast variation small-angle neutron scattering. \\ Journal of Applied Crystallography, 52 791-799.
}




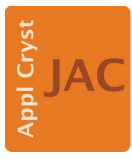

JOURNAL OF

APPLIED

CRYSTALLOGRAPHY

ISSN 1600-5767

Received 8 February 2019

Accepted 12 June 2019

Edited by G. Kostorz, ETH Zurich, Switzerland

Keywords: small-angle neutron scattering; polymer electrolyte fuel cells; catalysts; microstructure; water distribution.

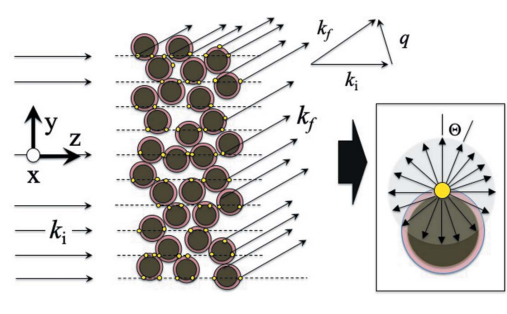

C 2019 International Union of Crystallography

\section{Microstructure and water distribution in catalysts for polymer electrolyte fuel cells, elucidated by contrast variation small-angle neutron scattering}

\author{
Satoshi Koizumi, ${ }^{a *}$ Satoru Ueda, ${ }^{a}$ Takumi Inada, ${ }^{a}$ Yohei Noda ${ }^{a}$ and Robert A. \\ Robinson $^{\mathrm{a}, \mathrm{b}}$
}

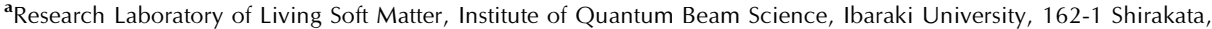
Tokai, Ibaraki 319-1106, Japan, and ${ }^{\mathbf{b}}$ School of Physics, University of Wollongong, Northfields Avenue, Wollongong, NSW 2522, Australia. *Correspondence e-mail: satoshi.koizumi.prof@vc.ibaraki.ac.jp
\end{abstract}

By using small-angle neutron scattering (SANS) reinforced by scanning electron microscopy, the fine structure of catalysts for polymer electrolyte fuel cells has been investigated. The experimental data resulting from contrast variation with mixed light and heavy water $\left(\mathrm{H}_{2} \mathrm{O} / \mathrm{D}_{2} \mathrm{O}\right)$ are well described by a core-shell model with fluctuations in concentration between water and Nafion. In particular, SANS obtained with the mixed water ratio 30/70, which corresponds to a matching point between mixed water and Nafion, shows a broad scattering maximum, which is attributed to a $5 \mathrm{~nm}$-thick Nafion shell on the surface of the larger carbon particles. After swelling by water, the ionomer layer absorbs water at the $17 \mathrm{wt} \%$ level. By changing the $\mathrm{H}_{2} \mathrm{O} / \mathrm{D}_{2} \mathrm{O}$ ratio, it was further confirmed that the catalyst with the ionomer exhibits water repellence, whereas the bare catalyst without the ionomer is wetted by water. Because it is very difficult to extract more information, for instance regarding the $\mathrm{Pt}-\mathrm{Nafion}$ interactions, by means of small-angle scattering, reflectometry and grazing-incidence scattering experiments with neutrons should be attempted on a model catalyst prepared on a flat substrate.

\section{Introduction}

In the past decade, polymer electrolyte fuel cells (PEFCs) have been commercialized for vehicle and residential usage (Wang et al., 2011). They are advantageous for high-voltage operation at low temperature, achieved by supplying hydrogen gas at the anode and oxygen gas at the cathode. As a result of power generation, water is produced according to the chemical reaction $\mathrm{H}_{2}+\mathrm{O}_{2} \rightarrow \mathrm{H}_{2} \mathrm{O}$, with a theoretical cell potential of $1.23 \mathrm{~V}$ (O'Hayre et al., 2006). In practice, there are three losses when we operate a PEFC at a certain current: (i) activation loss, (ii) resistance loss and (iii) diffusion loss. Diffusion loss in particular becomes dominant at high current operation. It is thought that diffusion loss occurs when generated water overflows and hinders oxygen gas diffusion at the surface of the cathode catalyst $(\mathrm{Pt})$. To overcome this issue, we need to control the microstructure in the vicinity of the platinum $(\mathrm{Pt})$ catalyst and the water distribution therein. The Pt catalyst comprises Pt particles of nanometre diameter, implanted on the surface of carbon particles whose diameter is of the order of $10 \mathrm{~nm}$. The surface of the Pt catalyst is covered with a thin ionomer layer in order to control proton conductivity in the thin layer. The essence of the physicochemical phenomena is the transport of multiple components (electrons, oxygen gas and protons) at the triple-phase contact interface near the Pt surface. This process has been intensively 
investigated for the perfluorinated sulfonic acid ionomers (Kusoglu \& Weber, 2017).

In this study, we investigate the microstructure of a catalyst ( $\mathrm{Pt}$ implanted on carbon powder) that is bound and coated by the ionomer Nafion. Small-angle X-ray and neutron scattering (SAXS and SANS) have been used many times before to study the microstructure of pure Nafion, both in the bulk and in films (Gebel \& Diat, 2005), and in this study we extend this work in order to understand the Nafion thin film on the Pt catalyst. Studies on Nafion thin films using neutron reflectivity have been reported by Wood et al. (2009), Eastman et al. (2012) and DeCaluwe et al. (2018), whereas in this study we intend to acquire information by SANS with contrast variation (Imae et al., 2011) in order to answer the question, what is the water distribution in the ionomer thin layer of the catalyst. Neutron scattering is sensitive to light atoms such as hydrogen and is suitable for in situ observation of the fuel cell during operation (Iwase et al., 2009; Putra et al., 2010; Ueda et al., 2019; Koizumi, Ueda, Putra \& Tsutsumi, 2019). Contrast variation was done by partially or fully exchanging water with deuterated water $\left(\mathrm{D}_{2} \mathrm{O}\right)$. To cover a wide range of observation length scale from ångströms to micrometres, we utilized ultrasmall-angle neutron scattering (USANS) with monochromatic neutrons, which allows access to ultra-small $q$, and time-offlight SANS with pulsed non-monochromatic neutrons, which allows access to higher $q$ (where $q$ is the wavenumber of the scattered neutrons). In order to reinforce our understanding of contrast variation SANS, we combine it with a complementary method, scanning electron microscopy (SEM). An image of secondary electrons (SEs) detected by SEM is sensitive to the external edge of the microstructure of the catalyst, which is referred to as the 'edge contrast effect'. In particular, the brightness of the SE image is quantitatively related to the projection image of the microstructures (Koizumi, Ueda, Nishikawa et al., 2019). Consequently, a twodimensional Fourier transform of the SE image corresponds to the structure and/or form factors of the microstructures, which are detected by the scattering method in reciprocal space.

As a result of observations by contrast variation SANS and SEM, we find for the fully humidified catalyst that (i) the thickness of the ionomer thin layer is about $5 \mathrm{~nm}$, (ii) the average water content is about $17 \mathrm{wt} \%$, and (iii) the layer is surrounded by air (void). Further information, such as the water distribution on the surface of the Pt or carbon or the microphase separation in the thin layer, can be achieved by grazing-incidence SANS (GISANS) including reflectivity, as reported by Ueda et al. (2018).

\section{Experimental}

\subsection{Small-angle neutron scattering spectrometry}

We utilized the time-of-flight SANS spectrometer iMATERIA at the Materials and Life Science Facilities (MLF), at the spallation neutron source J-PARC, Tokai, Japan. A wide band of wavelength $(\lambda)$ from 1 to $10 \AA$ is used to cover a wide $q$ region from $3 \times 10^{-3}$ to $40 \AA^{-1}$. The
iMATERIA spectrometer at BL20 was originally designed for powder diffraction, but was recently modified and upgraded in order to perform SANS measurements (Koizumi \& Noda, 2019). The versatile combination of SANS and powder diffraction allows wide- $q$ observation with high resolution. In this study, we select part of the $q$ region from 0.01 to $0.5 \AA^{-1}$, covered by small-angle and low-angle detector blocks, which highlights the microstructure of the catalyst.

To cover the ultra-small-angle scattering regime corresponding to micrometre length scales, we also utilized the SANS-J-II and PNO instruments at the JRR-3 research reactor, in Tokai, Japan. The 'focusing and polarized neutron ultra-small-angle scattering spectrometer' referred to as SANS-J-II was developed by the authors from 2000 to 2004 (Koizumi et al., 2006, 2007; Iwase et al., 2007). Using neutron focusing lenses (both magnetic and compound refractive), SANS-J-II can reach to the order of $10^{-4} \AA^{-1}$ by filling a missing $q$ region. Note that conventional SANS is usually limited to $q_{\min } \simeq 10^{-3} \AA^{-1}$. The $q$ range was further extended to a $q_{\text {min }}$ of $10^{-5} \AA^{-1}$, using the PNO Bonse-Hart doublecrystal USANS instrument (Yamaguchi et al., 2006).

\subsection{Scanning electron microscopy}

In order to obtain SE images of the catalyst with various magnifications, we employed a thermal field-emission scanning electron microscope (JSM-7800F, JEOL Co. Ltd) with resolutions of $0.8 \mathrm{~nm}(15 \mathrm{kV})$ and $1.2 \mathrm{~nm}(1 \mathrm{kV})$. The $\mathrm{SE}$ image is calibrated by studying monodisperse $\mathrm{SiO}_{2}$ particles by 2D Fourier transform according to the method reported by Koizumi, Ueda, Nishikawa et al. (2019). After brightness inversion and noise subtraction, the SE images were converted to the spectra in reciprocal space, which will be quantitatively compared with SANS data.

\subsection{Sample specimen}

In this study, we examine (i) the 'real catalyst' (catalyst powder bound by ionomer) and (ii) the neat catalyst powder (as a control). We also investigate a bulk film of Nafion (50 $\mu \mathrm{m}$ thickness) sandwiched by two catalyst layers, which we refer to as 'real MEA' (membrane electrode assembly). The preparation method of the catalyst layer is described below. Firstly, we prepared a mixture of Pt/C catalysts (Ketjen black, provided by TANAKA HOLDINGS Co. Ltd) and pure water with a mixing ratio of $36 / 50 \%(w / w)$. After stirring the mixture for $1 \mathrm{~h}$, we added an ionomer ink solution (Nafion/isopropyl alcohol) $[5 / 95 \%(w / w)]$ purchased from Aldrich Co. Ltd. In total, the weight ratio is catalyst/ionomer/water 36/14/50. We sprayed the mixed solution on the substrate using an airbrush and kept it in an oven at $333 \mathrm{~K}$ for $5 \mathrm{~min}$. We repeated this procedure ten times to obtain a catalyst layer of $0.5 \mathrm{mg} \mathrm{cm}^{-2}$. It was dried in an oven at $352 \mathrm{~K}$ for $1 \mathrm{~h}$. When we prepared the MEA, we pressed the catalyst sheet with Nafion film at $373 \mathrm{~K}$. For contrast variation SANS experiments, the sample specimens were swollen with mixed water $\left(\mathrm{H}_{2} \mathrm{O} / \mathrm{D}_{2} \mathrm{O}\right)$, by immersing the films in water overnight. 


\section{Experimental results}

\subsection{Scanning electron microscopy}

Fig. 1 shows the SE image of a section view of the MEA $(1000 \times)$ obtained after fuel-cell operation. We see the Nafion film at the bottom and at the top the catalyst (we refer to this as the 'real catalyst' in this paper). After use by fuel-cell operation, grooves are formed by the gas diffusion layer pressing on the catalyst.

Fig. 2 shows SE images for the real catalyst layer with different magnifications $(5000 \times, 100000 \times, 200000 \times$ and $500000 \times)$. The image with $100000 \times$ magnification shows individual primary carbon particles. With the highest magnification $(500000 \times)$, we can recognize $\mathrm{Pt}$ nano-particles implanted on the primary particles of carbon black. Although the particles are covered by the Nafion ionomer, the thin layer is not visible by electron microscopy.

Fig. 3 shows the two-dimensional Fourier transform of the SE images obtained for the real catalyst with different magnifications (shown in Fig. 2), i.e. the spectra are given as a function of wavenumber $q\left(\AA^{-1}\right)$ after circularly averaging at

Figure 1

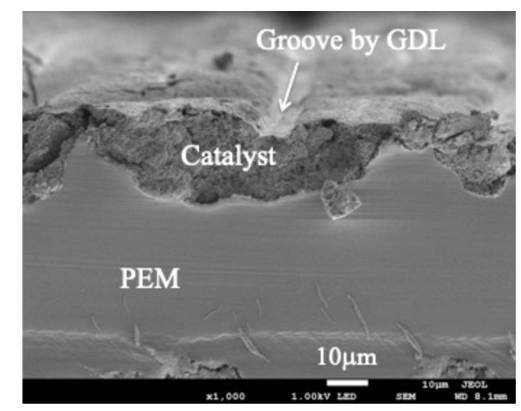

SE image of a cross section of the MEA after fuel-cell operation. At the bottom, the Nafion polymer electrolyte membrane (PEM), and at the top, the real catalyst ( $\mathrm{Pt} / \mathrm{C}$ catalyst mixed with ionomer binder).
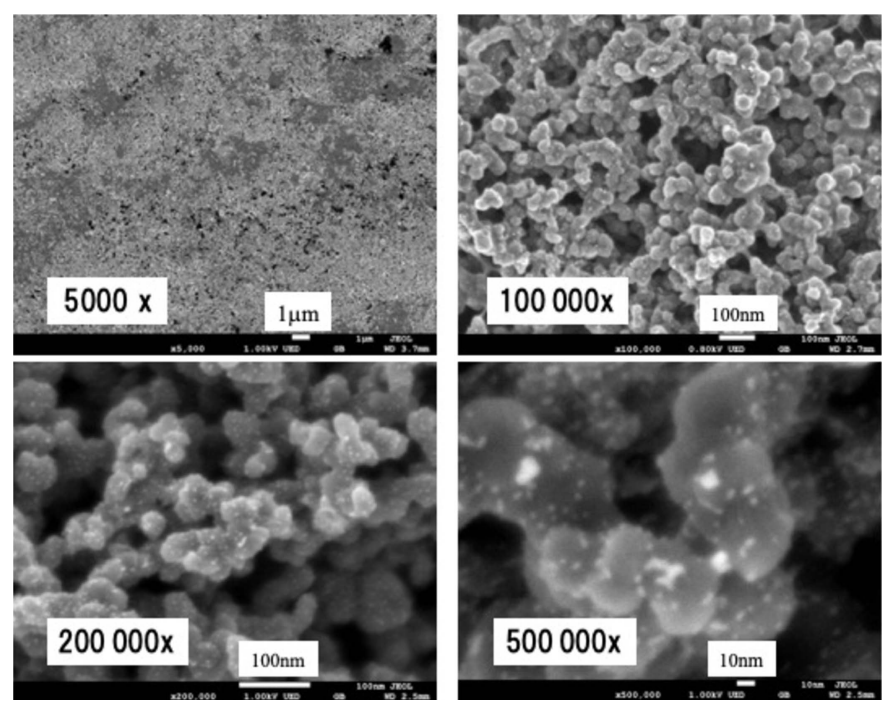

Figure 2

$\mathrm{SE}$ images obtained for the real catalyst ( $\mathrm{Pt} / \mathrm{C}$ catalyst mixed with ionomer binder), with varying magnifications: (a) $5000 \times$, (b) $100000 \times$, (c) $200000 \times,(d) 500000 \times$. $q=0$. Details of this method are given by Koizumi, Ueda, Nishikawa et al. (2019). The image with lower magnification appears at smaller $q$, and the individual spectra with different magnifications map onto a single master curve, which reflects the microstructure of the real catalyst. The upturn at lower $q$ is attributed to the interparticle interference (agglomerate) of the primary particles. At higher $q$ values, the two-dimensional Fourier transform of the SE image behaves according to $q^{-4}$ (Porod law originating from a sharp interface). This is due to the edge contrast effect of the SEs. There is a clear hump at $q=$ $0.01 \AA^{-1}$, originating from the primary particle of the catalyst powder. At higher $q$ for individual spectra, we recognize a plateau deviating from $q^{-4}$ behavior, which is attributed to the pixel resolution of the $\mathrm{SE}$ image $(\sim \mathrm{nm})$.

\subsection{Small-angle neutron scattering with contrast variation}

Fig. 4 shows SANS and USANS data obtained for the MEA after fuel-cell operation (corresponding to Fig. 1) by using PNO and SANS-J-II at JRR-3. The scattering curve covers a wide $q$ region from $10^{-5}$ to $1.0 \AA^{-1}$, corresponding to the length scales from $10 \mu \mathrm{m}$ to $1 \AA$ in real space. There is a $q^{-4}$ dependence in the $q$ region $10^{-4}<q<10^{-3}$, which originates from the surface structure of the agglomerate.

At higher $q$, with deviation from the asymptotic $q^{-4}$ behavior, the membrane of Nafion causes small-angle scattering. We found that the scattering maximum near $q=1.5 \AA^{-1}$ is due to an ion cluster swollen by $\mathrm{H}_{2} \mathrm{O}$ or dried. At $q=1.0 \AA^{-1}$, the crystalline order of Nafion gives rise to a scattering maximum.

The Fourier transform of the SE image in Fig. 3 will be compared with the $q$ behavior of SANS, in the region indicated by the rectangle in Fig. 4. For small $q$ values giving USANS or SANS and corresponding to the length scale of micrometres to $10 \mathrm{~nm}$ in real space, the microstructure in the catalyst is averaged according to the continuum approximation. Therefore, the 2D Fourier transform of the SE image,

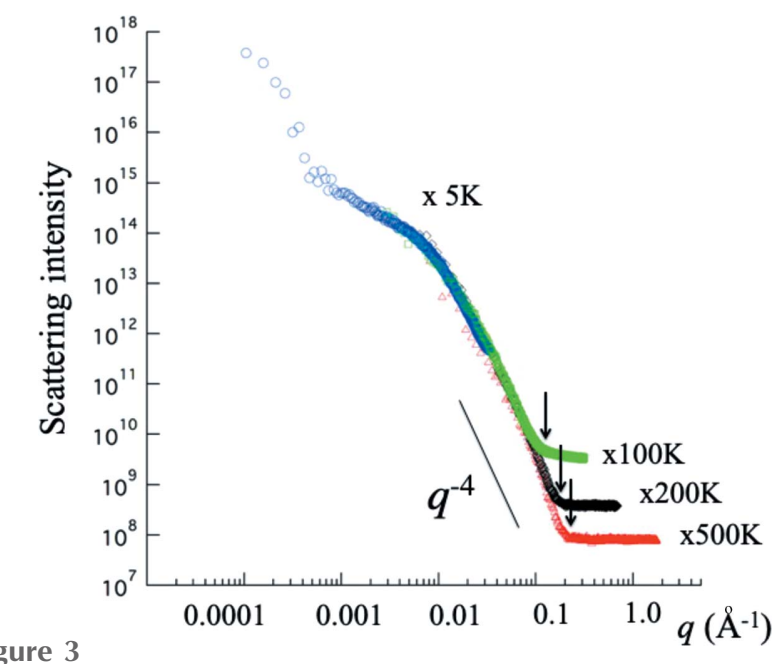

Figure 3

Two-dimensional Fourier transform of the SE images obtained for the real catalyst with the different magnifications shown in Fig. 2. Arrows show $q$ limitations due to the pixel resolution of the scanning electron microscope. The line is a guide showing $q^{-4}$ behavior. 
which is characteristic for the edge contrast effect, agrees well with USANS at this length-scale limit (Koizumi, Ueda, Nishikawa et al., 2019). In the high- $q$ region, corresponding to nanometre length scale, SANS detects not only the external shape of the microstructure but also the internal structure of

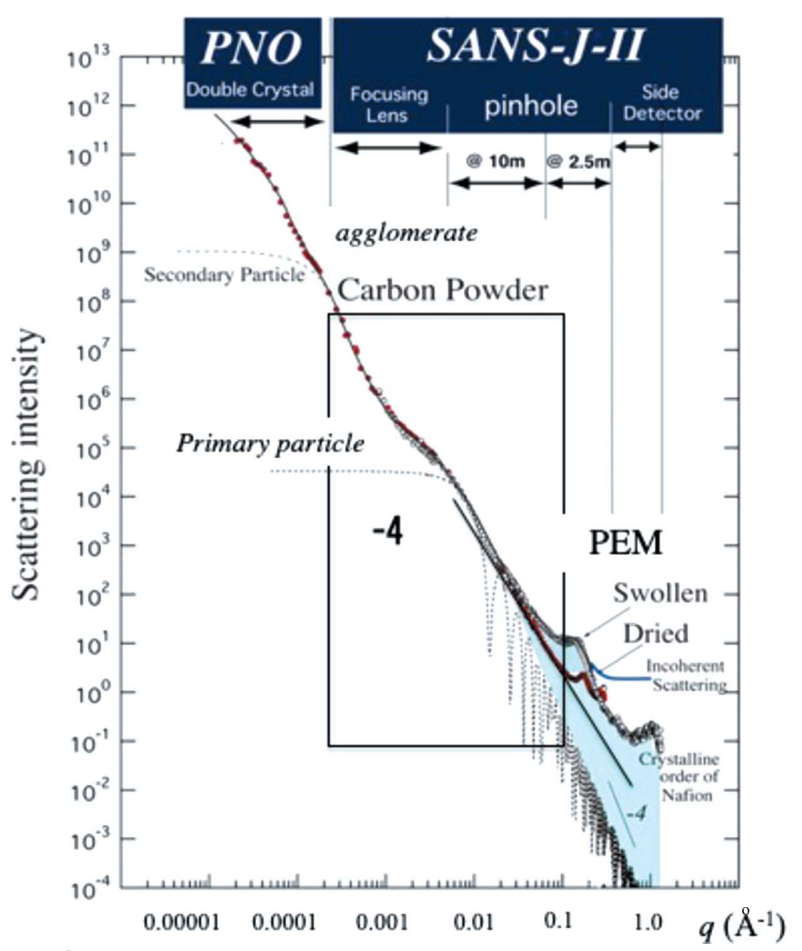

Figure 4

SANS and USANS obtained by the PNO and SANS-J-II spectrometers, for the real MEA specimen in states swollen by $\mathrm{H}_{2} \mathrm{O}$ or dried. The dotted curve indicates the form factor of a spherical domain with $r=15 \mathrm{~nm}$. The rectangle indicates the $q$ region corresponding to those shown in Figs. 3 and 5 .

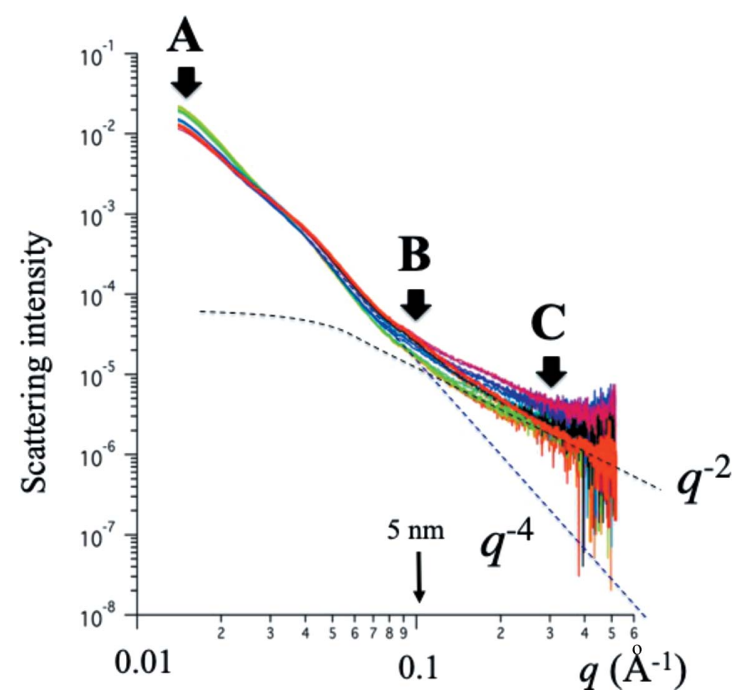

Figure 5

Contrast variation SANS obtained for the real catalyst with different mixing ratios of $\mathrm{H}_{2} \mathrm{O} / \mathrm{D}_{2} \mathrm{O}$. At different $q$ values, indicated by A, B and $\mathrm{C}$, we evaluate the scattering intensity as a function of $\mathrm{D}_{2} \mathrm{O}$ fraction in Fig. 7(a). The broken lines are a guide showing $q^{-2}$ and $q^{-4}$ behaviors. the catalyst. Therefore, the SANS data do not agree with the 2D Fourier transform of the SE image.

Fig. 5 shows SANS data obtained for the real catalyst by using the iMATERIA spectrometer. The observed $q$ region from 0.01 to $0.5 \AA^{-1}$ corresponds to the size of the primary particle or smaller than it. By changing the $\mathrm{H}_{2} \mathrm{O} / \mathrm{D}_{2} \mathrm{O}$ mixing ratio, we performed contrast variation measurements. SANS for low $q\left(<0.1 \AA^{-1}\right)$ is less sensitive to the mixing ratio, whereas at high $q\left(>0.1 \AA^{-1}\right)$, the SANS starts to deviate from $q^{-4}$ behavior and varies depending on the $\mathrm{H}_{2} \mathrm{O} / \mathrm{D}_{2} \mathrm{O}$ mixing ratio. Note that the $q$ value of $q=0.1 \AA^{-1}$ corresponds to length $\Lambda=6 \mathrm{~nm}$ in real space. This implies that the ionomer thin layer swollen by water gives rise to additional fluctuations in concentration. As for contrast variation by exchanging water $\left(\mathrm{H}_{2} \mathrm{O} / \mathrm{D}_{2} \mathrm{O}\right)$, the scattering intensity at low $q$ decreases as the $\mathrm{D}_{2} \mathrm{O}$ content increases. As the $\mathrm{D}_{2} \mathrm{O}$ content increases, the USANS intensity increases.

We also performed contrast variation SANS on the powder catalyst without the ionomer thin layer (Fig. 6). The SANS $q$ profiles change significantly as the $\mathrm{D}_{2} \mathrm{O}$ content increases, and the plateau is due to incoherent scattering from hydrogen in the light water $\left(\mathrm{H}_{2} \mathrm{O}\right)$.

Fig. 7 shows the scattering intensity at different $q$ values, as indicated by $\mathrm{A}, \mathrm{B}$ and $\mathrm{C}$ with thick arrows in Figs. 5 and 6. Fig. 7(a) shows the results for the real catalyst (with ionomer). At $q=0.01 \AA^{-1}$, the scattering intensity is almost constant (slightly increases at lower $\mathrm{D}_{2} \mathrm{O}$ content). At $q=0.1 \AA^{-1}$, the intensity shows a minimum at mixing ratios of $30 / 70$ and $20 / 80$. At $q=0.3 \AA^{-1}$, the decreasing tendency becomes more obvious and the minimum appears near 20/80.

As a control, we also examined the catalyst powder (without ionomer) [Fig. 7(b)]. At $q=0.01 \AA^{-1}$, the scattering intensity decreases monotonically as the $\mathrm{D}_{2} \mathrm{O}$ concentration increases. Note that a factor of $1 / 100$ is determined for the change between $0 / 100$ and $100 / 0$. At $q=0.1$ and $0.3 \AA^{-1}$, the

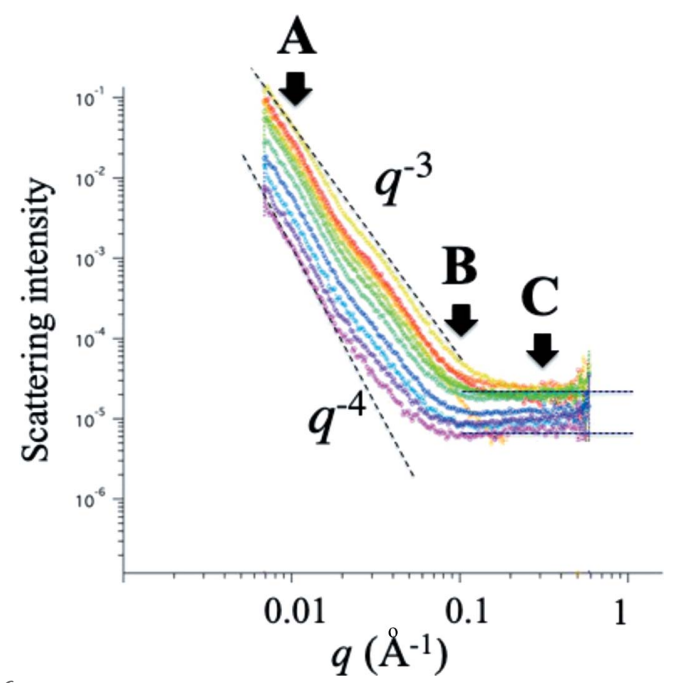

Figure 6

Contrast variation SANS obtained for the catalyst powder (no ionomer) with different mixing ratios of $\mathrm{H}_{2} \mathrm{O} / \mathrm{D}_{2} \mathrm{O}$. At different $q$ values, indicated by $\mathrm{A}, \mathrm{B}$ and $\mathrm{C}$, we evaluate the scattering intensity as a function of $\mathrm{D}_{2} \mathrm{O}$ fraction in Fig. $7(b)$. The broken lines are a guide showing $q^{-3}$ and $q^{-4}$ behaviors. 
intensity gradually decreases monotonically (incoherent scattering from hydrogen). The reason for these changes is later discussed with the analytical SANS model shown in Section 4.

\section{Analytical model for SANS}

In order to evaluate contrast variation SANS quantitatively, we introduce a structural model for the real catalyst as follows. We simply employ a core-shell model, in which the core (a primary particle of carbon) is covered with a shell (an ionomer thin layer swollen by water). In Fig. 8, as in our model, a single catalyst particle is dispersed in a matrix of air (or void). We suppose that the shell (ionomer thin layer) is swollen by water, causing fluctuations in concentration between the Nafion chains and water with an amplitude of $\Delta b_{3}$. The core and shell, with diameter $2 r$ and $2(r+\Delta r)$, respectively, have contrasts $\Delta b_{1}$ and $\Delta b_{2}$, as shown in Fig. 8.

Fig. 9 shows the scattering length density $(b)$ calculated for the individual scattering components: (1) void, (2) ionomer, (3) carbon and (4) mixed water. The thick straight lines indicate $b$ for (i) the void, (ii) a mixture of ionomer and mixed water, and (iii) carbon. The scattering length density $\left(b_{\mathrm{av}}\right)$ for the mixture of ionomer and mixed water is evaluated by considering a mixing ratio $\alpha$ :

$$
b_{\mathrm{av}}=(1-\alpha) b_{\mathrm{Nafion}}+\alpha\left[\phi b_{\mathrm{D} 2 \mathrm{O}}+(1-\phi) b_{\mathrm{H} 2 \mathrm{O}}\right] .
$$

The mixing ratio $\alpha$ will be determined as $\alpha=0.17$ later in Fig. 10, by considering the $q$ profile for the real catalyst swollen by mixed water $\mathrm{H}_{2} \mathrm{O} / \mathrm{D}_{2} \mathrm{O}(20 / 80)$.

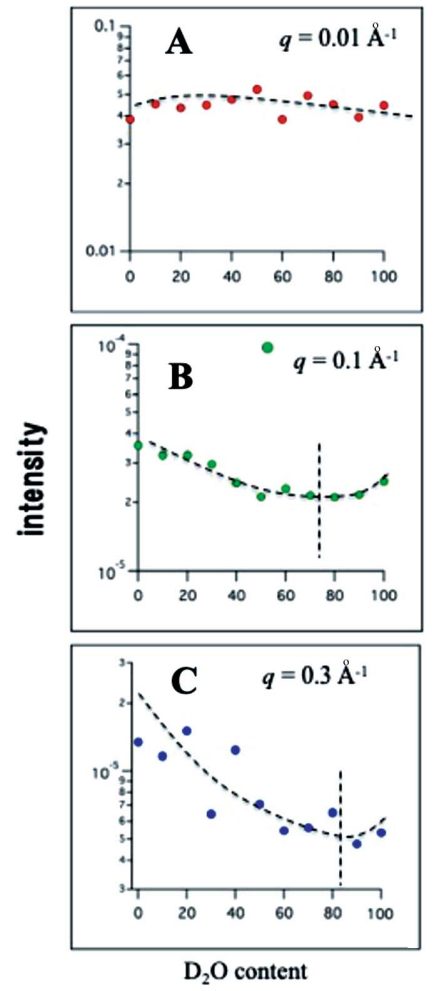

(a)
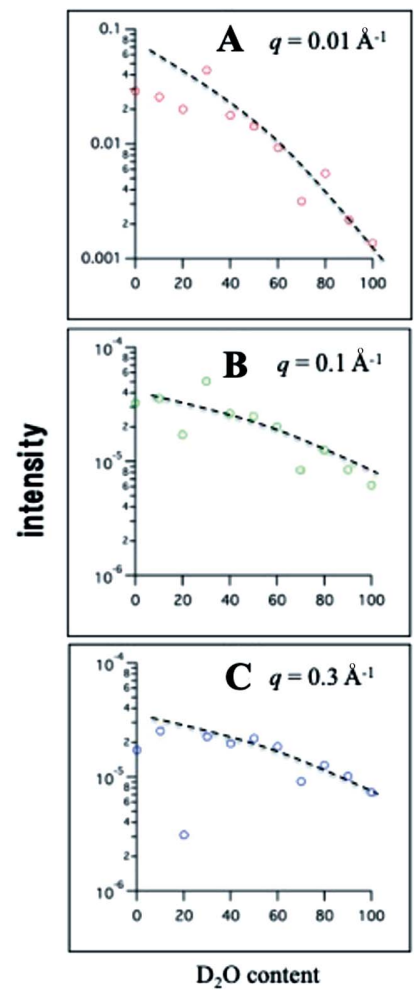

(b)
Figure 7

Scattering intensity evaluated at the different $q$ positions $\mathrm{A}, \mathrm{B}$ and $\mathrm{C}$ indicated in Figs. 5 and 6, $(a)$ for the real catalyst and $(b)$ for the catalyst powder. The broken lines are a guide for the eye.
The contrasts $\Delta b_{1}, \Delta b_{2}$ and $\Delta b_{3}$ are indicated in Fig. 9. $\Delta b_{1}$ and $\Delta b_{2}$ denote the scattering contrasts (differences in scattering length density) between the carbon powder and the ionomer thin layer, and between the ionomer thin layer and the matrix (void). They are defined as $\Delta b_{1}=b_{\text {ionomer }}-b_{\text {air }}$ and $\Delta b_{2}=b_{\text {carbon }}-b_{\text {ionomer }}$, where $b_{\text {carbon }}, b_{\text {ionomer }}$ and $b_{\text {air }}(=6.35$, 4.75 and $0 \times 10^{-6} \AA^{-2}$ ) are the scattering length densities for carbon, ionomer and mixed water, respectively. Note that when exchanging water with $\mathrm{D}_{2} \mathrm{O}$, the scattering densities $\Delta b_{1}$ and $\Delta b_{2}$ vary, because the ionomer thin layer is swollen by water.

Next we introduce a scattering function for describing the picture shown in Fig. 8. In addition to a core-shell model, we consider the concentration fluctuations in the shell and
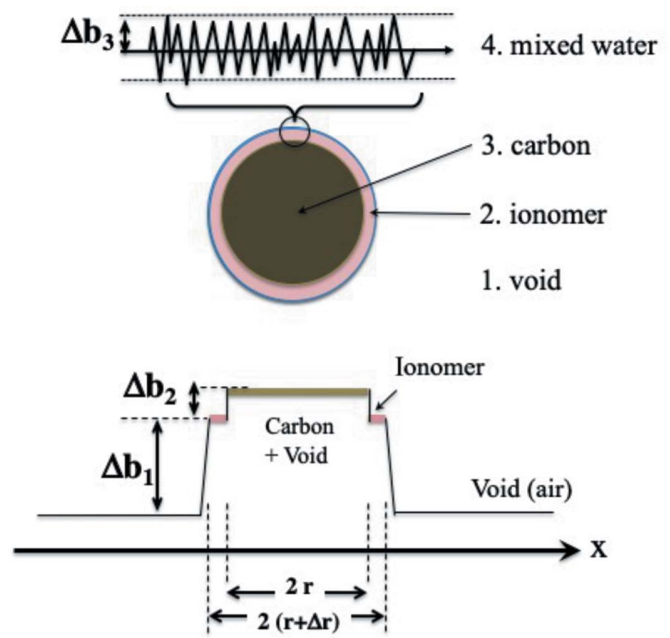

Figure 8

Structural model for SANS analysis, i.e. a core-shell model reproducing a primary particle of the real catalyst, in which the core (radius $r$ ) is carbon powder and the shell (thickness $\Delta r$ ) is an ionomer thin layer swollen by water. Scattering contrasts $\Delta b_{1}, \Delta b_{2}$ and $\Delta b_{3}$ are indicated.

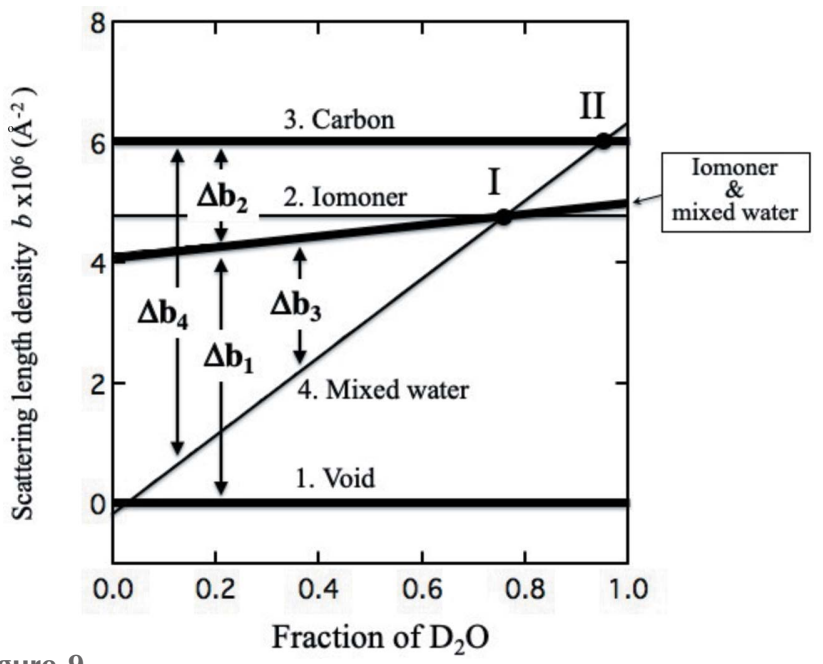

Figure 9

Scattering length density $(b)$ for the constituents of the real catalyst (1. ionomer, 2. carbon, 3. mixed water and 4. void) estimated as a function of $\mathrm{D}_{2} \mathrm{O}$ fraction. For the shell, $b$ for the ionomer and mixed water is also indicated. Scattering contrasts $\Delta b_{1}, \Delta b_{2}, \Delta b_{3}$ and $\Delta b_{4}$ are indicated with arrows. 
incoherent scattering from hydrogen, $I_{\text {inc. The scattering }}$ function for the real catalyst is given by

$$
I(q) \propto L(q)\left[\Delta b_{1} f(q, r+\Delta r)+\Delta b_{2} f(q, r)\right]^{2}+C(q)+I_{\mathrm{inc}},
$$

where $f(q, r)$ is the scattering amplitude for a spherical domain. $f(q, r)$ is given by

$$
f(q, r)=\frac{3}{q^{3} r^{3}}[\sin (q r)-q r \cos (q r)] .
$$

The second term in equation (2), $C(q)$, describes the scattering function for the local concentration fluctuations in the shell. By postulating that the distribution of water in the shell is homogeneous, we obtain

$$
C(q)=C \frac{\Delta b_{3}^{2}}{1+(q \xi)^{2}},
$$

where $\xi$ is the correlation length for local concentration fluctuations, and $\Delta b_{3}$ is the difference in scattering length density between the ionomer and mixed water. $L(q)$ plays a role in the USANS region, describing agglomeration of primary particles:

$$
L(q) \simeq q^{-\beta}
$$

If $r \gg \Delta r(\cong 0)$, we can approximate as follows:

$$
\begin{aligned}
I(q) \cong & L(q)\left[\left(\Delta b_{1}^{2}+\Delta b_{2}^{2}\right) f(q, r)^{2}\right. \\
& \left.+2 \Delta b_{1} \Delta b_{2} f(q, r) f(q, r+\Delta r)\right]+C(q)+I_{\mathrm{inc}}
\end{aligned}
$$

In equation (6), the first term, with an asymptotic decay of $q^{-4}$, should be identical to the Fourier transform of the SE image; the second and third terms, which cannot be detected by electron microscopy, are deviations from the continuum approximation.

For high $q\left(>0.1 \AA^{-1}\right)$, corresponding to a length scale $\Lambda \sim$ $\mathrm{nm}, I(q)$ is approximated as follows:

$$
I(q) \cong 2 \Delta b_{1} \Delta b_{2} f(q, r) f(q, r+\Delta r)+C(q)+I_{\text {inc }} .
$$

In the region $q<0.1 \AA^{-1}$, the form factor for the primary carbon particle is dominant, as given by

$$
I(q) \cong L(q)\left(\Delta b_{1}^{2}+\Delta b_{2}^{2}\right) f(q, r)^{2} .
$$

In the USANS $q$ region, corresponding to length scales $\Lambda \sim$ $\mu \mathrm{m}, f(q, r) \cong f(q, r+\Delta r)=1$. In this $q$ region, the agglomerate is dominant. Therefore, we obtain

$$
I(q) \cong\left(\Delta b_{1}^{2}+\Delta b_{2}^{2}\right) L(q) .
$$

This limit can be observed in the $q$ region of $10^{-5} \AA^{-1}$.

\section{Discussion}

\subsection{Microstructure of catalyst and swelling behavior}

As a result of the comparison between SANS and electron microscopy, we confirmed that the structural information regarding the ionomer thin layer appears in the high- $q$ region at around $q=0.1-0.5 \AA^{-1}$, where we see a deviation from the $q^{-4}$ asymptotic behavior. In this $q$ region, three factors (shell of thin layer, concentration fluctuations in the shell and incoherent scattering from hydrogen) determine the SANS intensity and its $q$ behavior. For smaller $q$, on the other hand, the primary particle of the carbon catalyst dominates both SANS and USANS.

By considering a structural model, we discuss Fig. 7 more carefully.

(i) According to Fig. 7(a)-A for the real catalyst, the scattering intensity at $q=0.01 \AA^{-1}$ is almost constant. This means that the carbon powder is surrounded by a void because water is repelled by the ionomer coating. Therefore, surface coating with the ionomer Nafion allows a fuel gas diffusion path to be established.

(ii) On the other hand, for the catalyst powder [Fig. 7(b)-A], the scattering intensity at $q=0.01 \AA^{-1}$ monotonically decreases as $\mathrm{D}_{2} \mathrm{O}$ content increases. This decrease by a factor of $1 / 100$ is due to $b_{\mathrm{av}}^{2} \sim(1-\phi)^{2}$ in equation (1). This in turn is due to the contrast $\Delta b_{4}$, implying that the catalyst is surrounded by water so the contrast $\Delta b_{4}$ decreases as the $\mathrm{D}_{2} \mathrm{O}$ content increases.

(iii) For Figs. 7(a)-B and 7(a)-C, the minimum of intensity appears around the mixing ratio $\mathrm{H}_{2} \mathrm{O} / \mathrm{D}_{2} \mathrm{O}=20 / 80$. According to Fig. 9, the mixing ratio $\mathrm{H}_{2} \mathrm{O} / \mathrm{D}_{2} \mathrm{O}=20 / 80$ is close to the matching point in contrast between the ionomer (Nafion) and water. Therefore, $C(q)=0$ and the structure factor of the catalyst becomes more obvious in the $q$ behavior.

(iv) The correlation length $\xi$ of $C(q)$ is of the order of $\mathrm{nm}$.

(v) In Figs. 7(b)-B and 7(b)-C, incoherent scattering $I_{\text {inc }}$ from hydrogen is dominant.

Next, we evaluate SANS obtained for the real catalyst swollen by mixed water $\left(\mathrm{H}_{2} \mathrm{O} / \mathrm{D}_{2} \mathrm{O} 30 / 70\right)$. In Fig. 10, the SANS curves marked $(a)$ and $(b)$ were obtained by the SANSJ-II and iMATERIA spectrometers, respectively. The curve marked (c) (black line) is calculated by equations (6) and (7) with the scattering contrasts $\Delta b_{1}=4.75 \times 10^{6} \AA^{-2}$ and

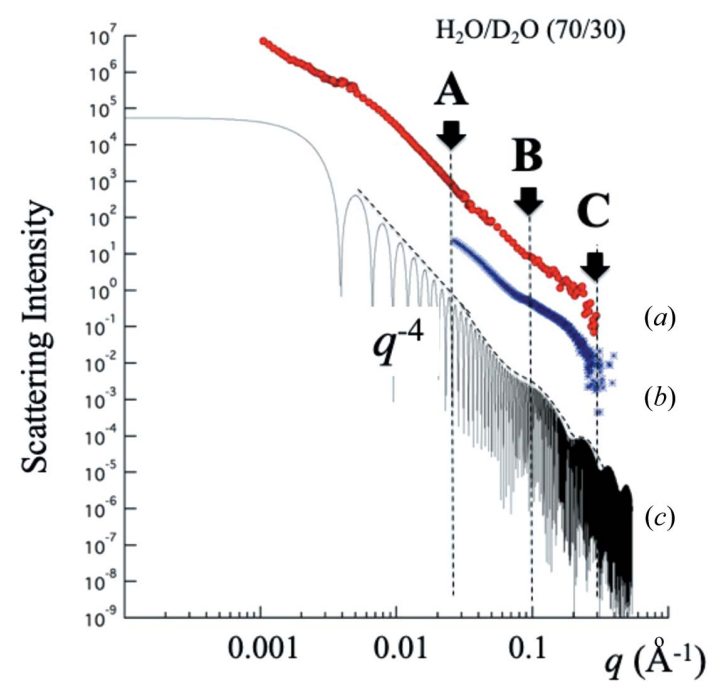

Figure 10

SANS curves for the real catalyst with mixed water $\mathrm{H}_{2} \mathrm{O} / \mathrm{D}_{2} \mathrm{O}(30 / 70)[(a)$ and $(b)$ obtained by SANS-J-II and iMATERIA spectrometers]. The scattering curve $(c)$ was obtained by model analysis based on a core-shell model, reproducing a primary particle of the real catalyst with $r=12 \mathrm{~nm}$, $\Delta r=5 \mathrm{~nm}$ and $\alpha=17 \mathrm{wt} \%$. Note that this curve has not been smeared by the instrumental resolution function. 
$\Delta b_{2}=1.5 \times 10^{6} \AA^{-2}$, and with sizes of $r=12 \mathrm{~nm}$ and $\Delta r=$ $5 \mathrm{~nm}$ (the thickness of the ionomer layer and the radius of the primary particle). The calculated curve $(c)$ well reproduces the broad shoulder that appears at around $q=0.07-0.2 \AA^{-1}$, and its intensity is $10^{-6}$ lower as compared to the top of the form factor. In the region of $q<0.03 \AA^{-1}$, the asymptotic $q$ behavior is according to $q^{-4}$, whereas in the region of $0.03<q<$ $0.07 \AA^{-1}$, the asymptotic $q$ behavior becomes steeper than $q^{-4}$. By considering $\Delta b_{2}$, we determined the water content in the ionomer to be $\alpha=17 \mathrm{wt} \%$. It should be noted that the ionomer thickness of $5 \mathrm{~nm}$ is close to the mean distance between ion clusters formed in a bulk film (Gierke et al., 1981). When the mixing ratio deviates from the matching point (I indicated in Fig. 9), the concentration fluctuations related to $C(q)$ are observed again and the hump due to the core-shell structure becomes invisible.

5.2. Further information: where is the water in the ionomer layer?

SANS can be described by the Debye equation, assuming a random orientation of particles on average (see Appendix $A$ ). To perform more detailed structural analysis on local structures, for example, the distribution of water on the Pt or carbon surface in the catalyst, it is necessary to use an alternative method, namely GISANS including reflectivity. The reflectivity is especially sensitive to the mass distribution $(\mathrm{d} b /$ $\mathrm{d} z$ ) along the normal vector of the surface. For that purpose, in a previous study we simulated local structures of the catalyst on an Si substrate, by sputtering and spin-coating methods, and then performed GISANS and reflectivity measurements (Ueda et al., 2018).

By comparing with results obtained by the GISANS and reflectivity methods, we discuss the microphase structure and water distribution in the ionomer thin layer. Ueda et al. (2018) performed GISANS and neutron reflectivity measurements on a model catalyst, i.e. the Nafion ionomer was spread by the spin-coating method on platinum and silicon substrates with thickness $10 \mathrm{~nm}$. During evaporation of the solvent, the ionomer orders under the constraint of the thin and narrow space. Affinity with the top and bottom interfaces also affects the ordering. As a result of GISANS and neutron reflectivity measurements, we concluded that (i) there is no microphase separation (ion cluster) in the ionomer thin layer $(10 \mathrm{~nm}$ thickness). The results from neutron reflectivity further indicated that (ii) the water content is $\sim 17 \mathrm{wt} \%$ in the ionomer thin layer (10 $\mathrm{nm}$ thickness) on the Pt surface, and (iii) on the platinum surface, the water preferentially attaches with a thickness of $0.3 \mathrm{~nm}$.

5.2.1. Research on model catalysts on a substrate. Model catalysts prepared on a flat substrate have been widely investigated. By using atomic force microscopy coupled with the electrochemical method (e-AFM) and contact angle measurements, Ohira et al. (2013) examined the surface morphology and properties of proton conduction. The ionomer thin layer was prepared on different substrates (silicon, Pt, glassy carbon and hydrophobic carbon). It was reported that the surface morphology is strongly affected by the substrate and significantly different from that of the bulk thick film. It was also reported that, depending on the surface material, the hydrophobicity or hydrophilicity at the air interface was changed. By using positron annihilation coupled with complementary X-ray photoelectron spectroscopy and AFM, Mohamed et al. (2013) reported that a number of hydrophilic inverse clusters of $\mathrm{SO}_{3}{ }^{-}$are formed on the surface of the ionomer thin layer on $\mathrm{Si}$, but not on the other materials. Yagi et al. (2014) employed vibrational sum frequency generation spectroscopy in order to probe the interface between the ionomer and $\mathrm{Pt}(100)$. The preferential orientation of an $\mathrm{SO}_{3}{ }^{-}$group on the platinum oxide (PtO) surface was proposed, based on the results obtained by cyclic voltammetry and chronoamperometry using ultramicroelectrode electroimpedance spectroscopy (Chlistunoff \& Pivovar, 2015). Affinity to water for a pendant side chain was examined by using an aqueous solution of perfluorooctanesulfonic acid, which is regarded as a model of a branch chain of Nafion (Inada et al., 2019).

5.2.2. Water-repellent effect. In this section, we consider a peculiarity of the hydrophobicity of Nafion. A Nafion film, which is composed of a polytetrafluoroethylene (PTFE) backbone, is well known as hydrophobic. A C $-\mathrm{F}$ bond, on the other hand, has a large dipole, which suggests to us that an individual molecule is both polar and hydrophilic. The inconsistency between the single molecule and the molecular assembly might be a crucial consideration regarding the water affinity of the Nafion thin layer in the catalyst.

Recently, a stratified dipole-array (SDA) model (Hasegawa et al., 2014) was proposed in order to explain the water affinity of PTFE. The peculiarity of perfluoroalkyl compounds is that they exhibit a hydrophobic behavior at their surface when they form a solid. Firstly, a PTFE chain is straight and helical, unlike an alkyl chain $\left(\mathrm{CH}_{2}\right)$, because of the larger electron cloud of the fluorine atom. In a bulk film with high crystalline order, the polarity found for a single molecule is completely canceled out, with packing in a two-dimensional aggregate of perfluoroalkyl (Rf) groups. This aggregate is spontaneously induced by dipole-dipole interaction arrays owing to the helical structure of the Rf group. It is reported that 'short' Rf molecules have hydrophilic character, whereas 'long' Rf molecules spontaneously form a hexagonal aggregate. This is a mechanism by which a polar chain can behave as hydrophobic, referred to as an SDA scenario (Hasegawa et al., 2014). In other words, the $\mathrm{CF}_{2}$ dipole is invisible in the crystalline symmetry.

Next, we turn to the ionomer thin layer of the catalyst. The crystalline order of the PTFE chains might be imperfect in the thin layer of $5 \mathrm{~nm}$ thickness. In the thin layer, about ten PTFE chains (the diameter of a $\mathrm{CF}_{2}$ chain is $c a 0.4 \mathrm{~nm}$ ) are aligned along the film surface, together with pedant side chains. However, we found that the real catalyst repels water, forming a void surrounding the powder. Thus the SDA scenario established for bulk Nafion film is also effective for the thin Nafion layer in the catalyst. Contrast variation SANS also found that the thin layer is swollen by water. Especially 
around sulfonic acid groups, the water might be localized preferentially. The concentration fluctuations in the thin layer, giving the scattering contrast $\Delta b_{3}$, result in the scattering $C(q)$.

\section{Conclusion}

By combining observation methods in real and reciprocal space, SANS and SEM, we have investigated the microstructure of a catalyst for a fuel cell, especially focusing on the ionomer thin layer and water distribution. As a result of contrast variation SANS with mixed water $\left(\mathrm{H}_{2} \mathrm{O} / \mathrm{D}_{2} \mathrm{O}\right)$, it was found that, in a catalyst prepared according to a standard recipe, the Nafion ionomer covers the carbon-supported $\mathrm{Pt}$ with a thickness of $5 \mathrm{~nm}$. After swelling by water, the ionomer layer absorbs water at the $17 \mathrm{wt} \%$ level. Water repellence was also observed for the catalyst bound by the ionomer, whereas the catalyst powder without the ionomer is covered with water. Further developments on understanding of the local water distribution will be achieved by using GISANS and neutron reflectivity on model catalysts on flat substrates.

\section{APPENDIX $A$}

Scattering geometry for small-angle scattering and reflectivity

We define the sample coordinate system $(x, y, z)$ with the $z$ axis parallel to the normal vector of the film and the $x$ and $y$ axes parallel to the film surface. The scattering process is described by conservation laws for momentum transfer $\hbar \mathbf{q}=\left(\mathbf{k}_{\mathrm{f}}-\mathbf{k}_{\mathrm{i}}\right)$ and energy transfer $\hbar \omega=\left(\hbar^{2} / 2 m\right)\left(\mathbf{k}_{\mathrm{f}}^{2}-\mathbf{k}_{\mathrm{i}}^{2}\right)$, where $\hbar$ is Planck's constant, $\left|\mathbf{k}_{\mathrm{i}}\right|$ and $\left|\mathbf{k}_{\mathrm{f}}\right|$ are the wavenumbers of the neutron before and after scattering, and $m$ is the mass of the neutron. In general, for elastic scattering, $\left|\mathbf{k}_{\mathrm{i}}\right|=\left|\mathbf{k}_{\mathrm{f}}\right|=2 \pi / \lambda$. The scattering vector is given by $\mathbf{q}=\left(q_{x}, q_{y}, q_{z}\right)$, and the wavenumber $q$ is

$$
q=|\mathbf{q}|=(4 \pi / \lambda) \sin \theta
$$

where $\lambda$ and $2 \theta$ are the wavelength and scattering angle, respectively.

If SANS is performed with the incident beam along the $z$ axis then we obtain $\mathbf{q}=\left(q_{x}, q_{y}, 0\right)$. By circularly averaging in $q$, with the position of the direct neutrons as the origin, a SANS intensity $I(q)$ is acquired as a function of $q=\left(q_{x}^{2}+q_{y}^{2}\right)^{1 / 2} \cdot I(q)$ is given by

$$
I(q) \propto N\left\langle\left|\iint_{V} b(r, \Theta) \exp (i \mathbf{q} \cdot \mathbf{r}) \mathrm{d}^{3} \mathbf{r}\right|^{2}\right\rangle_{\Theta} .
$$

The catalyst powder is packed in a plate-shaped cuvette, i.e. an isotropic and spherically symmetric system (see Fig. 11). Averaging over the random orientation $\Theta$ gives the Debye formalism, as follows:

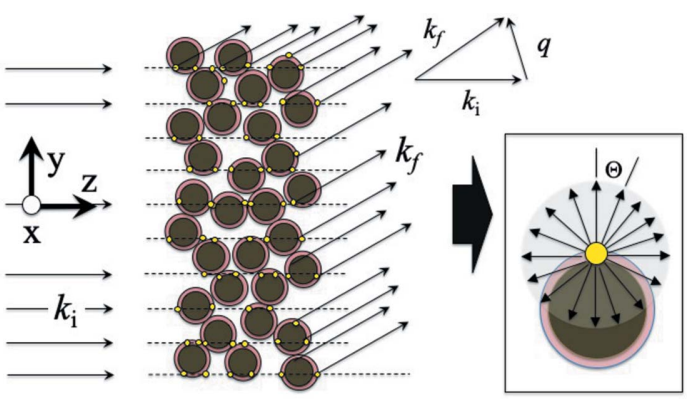

Figure 11

Schematic diagram showing a SANS experiment on a catalyst powder specimen, where core-shell ionomer/carbon and Pt particles (yellow) are depicted. The incident beam is along the $z$ axis, and SANS is detected in the $x y$ plane. In the beam, the power specimen is randomly oriented and therefore averaging over the orientation $\Theta$ gives the Debye formalism [equation 12] (see right inset).

$$
I(q) \propto(4 \pi)^{2}\left|\int_{0}^{R} r^{2} b(r) \frac{\sin (q r)}{(q r)} \mathrm{d} r\right|^{2} .
$$

In our small-angle scattering studies with real carbonplatinum-Nafion systems, the experimental results are well modeled with a core-shell model, with a $5 \mathrm{~nm}$-thick Nafion shell on the surface of the larger carbon particles and water content at the $17 \mathrm{wt} \%$ level. It is very difficult to extract more information, for instance, regarding the Pt-Nafion interactions, by means of small-angle scattering because of the random orientation of the $\mathbf{q}$ vectors (see the right of Fig. 11) and therefore equation (12). This situation leads us to attempt reflectometry and grazing-incidence scattering experiments with a specimen prepared on a flat substrate.

\section{Funding information}

This work was partially supported by the Research and Development of Polymer Electrolyte Fuel Cell Technology project of the New Energy and Industrial Technology Development Organization (NEDO), Japan.

\section{References}

Chlistunoff, J. \& Pivovar, B. (2015). J. Electrochem. Soc. 162, F890F900.

DeCaluwe, S. C., Bakerb, A. M., Bhargavab, P., Fischera, J. E. \& Durab, J. A. (2018). Nano Energy, 46, 91-100.

Eastman, S. A., Kim, S., Page, K. A., Rowe, B. W., Kang, S., Soles, C. L. \& Yager, K. G. (2012). Macromolecules, 45, 7920-7930.

Gebel, G. \& Diat, O. (2005). Fuel Cells, 5, 261-276.

Gierke, T. D., Munn, G. E. \& Wilson, F. C. J. (1981). J. Polym. Sci. Polym. Phys. Ed. 19, 1687-1704.

Hasegawa, T., Shimoaka, T., Shioya, N., Morita, K., Sonoyama, M., Takagi, T. \& Kanamori, T. (2014). ChemPlusChem, 79, 1421-1425. Imae, T., Kanaya, T., Furusaka, M. \& Torikai, N. (2011). Neutrons in Soft Matter. Hoboken: John Wiley \& Sons.

Inada, T., Maeda, T., Noda, Y. \& Koizumi, S. (2019). JPS Conf. Proc. 25, 011019. 
Iwase, H., Koizumi, S., Iikura, H., Matsubayashi, M., Yamaguchi, D., Maekawa, Y. \& Hashimoto, T. (2009). Nucl. Instrum. Methods Phys. Res. A, 605, 95-98.

Iwase, H., Koizumi, S., Suzuki, J., Oku, T., Sasao, H., Tanaka, H., Shimizu, H. M. \& Hashimoto, T. (2007). J. Appl. Cryst. 40, s414-s417.

Koizumi, S., Iwase, H., Suzuki, J., Oku, T., Motokawa, R., Sasao, H., Tanaka, H., Yamaguchi, D., Shimizu, H. M. \& Hashimoto, T. (2006). Physica B, 385-386, 1000-1006.

Koizumi, S., Iwase, H., Suzuki, J., Oku, T., Motokawa, R., Sasao, H., Tanaka, H., Yamaguchi, D., Shimizu, H. M. \& Hashimoto, T. (2007). J. Appl. Cryst. 40, s474-s479.

Koizumi, S. \& Noda, Y. (2019). JPS Conf. Proc. 25, 011004.

Koizumi, S., Ueda, S., Nishikawa, Y., Terao, T. \& Kubo, N. (2019). J. Appl. Cryst. 52, 783-790.

Koizumi, S., Ueda, S., Putra, A. \& Tsutsumi, Y. (2019). AIP Adv. 9, 065206.

Kusoglu, A. \& Weber, A. Z. (2017). Chem. Rev. 117, 987-1104.

Mohamed, H. F. M., Kuroda, S., Kobayashi, Y., Oshima, N., Suzuki, R. \& Ohira, A. (2013). Phys. Chem. Chem. Phys. 15, 1518-1525.
O’Hayre, R., Cha, S., Colella, W. \& Prinz, F. B. (2006). Fuel Cell Fundamentals. Hoboken: John Wiley \& Sons.

Ohira, A., Kuroda, S., Mohamed, H. F. M. \& Tavernier, B. (2013). Phys. Chem. Chem. Phys. 15, 11494-11500.

Putra, A., Iwase, H., Yamaguchi, D., Koizumi, S., Maekawa, Y., Matsubayashi, M. \& Hashimoto, T. J. (2010). J. Phys. Conf. Ser. 247, 012044.

Ueda, S., Koizumi, S., Ohira, A., Kuroda, S. \& Frielinghaus, H. (2018). Physica B, 551, 309-314.

Ueda, S., Koizumi, S. \& Tsutsumi, Y. (2019). Res. Phys. 12, 504511.

Wang, Y., Chen, K. S., Mishler, J., Cho, S. C. \& Adroher, X. C. (2011). Appl. Energy, 88, 981-1007.

Wood, D. L., Chlistunoff, J., Majewski, J. \& Botup, R. L. (2009). J. Am Chem. Soc. 50, 18096-18104.

Yagi, I., Inokuma, K., Kimijima, K. \& Notsu, H. J. (2014). J. Phys. Chem. C, 118, 26182-26190.

Yamaguchi, D., Koizumi, S., Motokawa, R., Kumada, T., Aizawa, K. \& Hashimoto, T. (2006). Physica B, 385-386, 1190-1193. 